\title{
Research on Electrical and Electronic Instructions Based on a Real-time Feedback System
}

\author{
Ping $\mathrm{Yu}^{\mathrm{a}}$, Feiqing $\mathrm{Wu}$, Enjun $\mathrm{Yu}$, Jiong Sun \\ Ningbo Institute of Technology, Zhejiang University, Ningbo 315100, China \\ ayuping@nit.zju.edu.cn
}

Keywords: Electrical and electronic courses, interactive instruction, classroom interactive feedback system.

\begin{abstract}
This paper reviews the feedback methods which a conventional electrical and electronic course usually employs. After analyzing the characteristics of the conventional electrical and electronic conventional classroom teaching, we find the asymmetry of feedback channels for the students and the instructor. In order to improve the teaching interaction of the electrical and electronic course, a mobile-web-based classroom interactive response system (CIRS) is proposed, designed and realized. The application of the proposed CIRS combined with smart phones suggests great potentials in promoting the students' understanding and inner construction of teaching contents.
\end{abstract}

\section{Introduction}

Modern information society requires that talents who graduate from universities of science and engineering must have the ability to understand the principles of electrical and electronics, to operate or maintain electronic equipment. Therefore, electrical and electronic courses are preliminary course for students who major in science and engineering. Commonly, the electrical and electronic courses include a sets of courses such as principles of electronic circuits, analog circuits, digital circuits and electrotechnics. However, as foundation courses, these courses sometimes are scheduled in a single semester. As for teachers, it is significant and challengeable to make these courses interesting and their instruction effective due to the reduced class hours. Various teaching methods and instructional technologies are introduced in order to improve the teaching of electrical and electronic courses [1-2].

In this work, we designed and realized a web-based classroom feedback system for electrical and electronic courses teaching. This system is used to enhance the interaction in a large-scale electronic theory lecture and these course are organized in a novel style of flipped classroom [3]. The system is designed to support mainstream smart mobile devices for the convenience of access.

\section{Analysis of instruction feedback in conventional electrical and electronics courses}

Electrical and electronic courses involve a series of concepts, theorems, analysis methods and application samples. In a traditional electronic lectures, these theories are introduced and demonstrated only by the teacher, and the main form of the students' learning is listening. But for a high-efficiency lecture, the teaching and learning activates in the classroom should be interactive. That is, effective feedback channels should be set up between the teacher and the students. Commonly, the feedbacks obtained by the instructor from the students include emotional, verbal and behavioral information. For example, question-response method can received a few representative feedbacks from several random students. The information extracted from these feedbacks sometimes include common troubles in the students' understanding and it would be helpful to the instructor's teaching.

These feedbacks in the forms of emotional, verbal and behavioral info are instant but not overall and difficult to distinguish [4], thus sometimes quiz is used to get more concrete feedbacks. However, due to the large scale of electronic courses and limited class time, the whole results of the quiz cannot be received and evaluated immediately. As a result, the feedback channel between the teacher and the 
students is asymmetric: the students get feedback easily from the teacher because of the teacher's "broadcast", but not the same to the teacher due to the large number of students.

\section{Design and realization of CIRS for electrical and electronic Courses}

From the above analysis, the lack of effective feedback channels from the students to the teacher is the main reason that an effective and interactive lecture cannot be carried out. Therefore, the issues on feedback channel must be resolved for constructing smooth interactive environment. Here, we propose a feedback scheme which would substantially improve the interaction mode between the teacher and the students. Figure 1 shows the schematic of the proposed interactive feedback configuration. The students can access the syllabus, lecture slides and other reference materials from the server through the wireless LAN (WLAN). They also can send the teacher feedbacks such as requesting lower speed, asking some questions or submitting a quiz simultaneously through their smart mobile devices. In the way as shown in Fig. 1, the teacher can obtain the exact status of the students' learning. For example, how many students have the same issues on understanding a knowledge point? Is there any request for a lower speech speed? Based on these received and analyzed feedbacks, the teacher could adjust their teaching methods and decide how to handle these matters correspondingly. The project screen also acts as an interactive wall, which shows the summary results of a quiz, or present the discussion of the students on a specific topic.

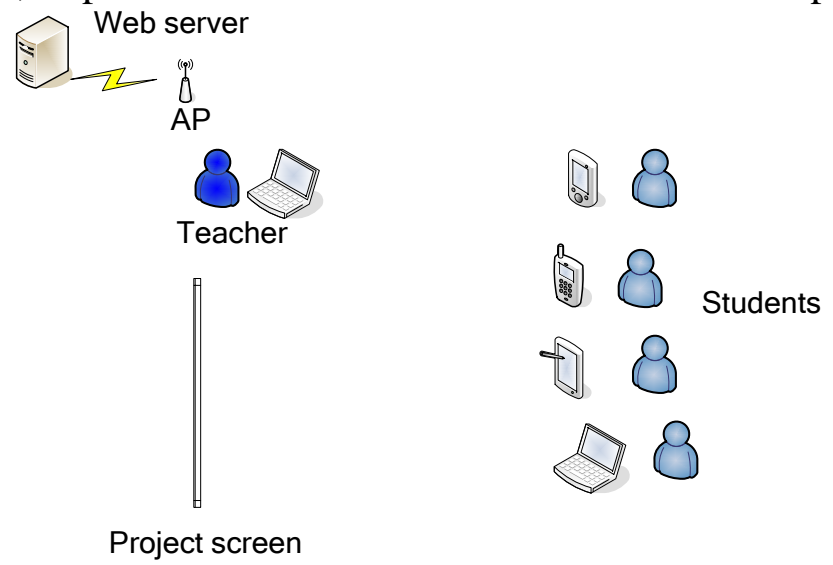

Fig. 1 schematic of the interactive feedback classroom

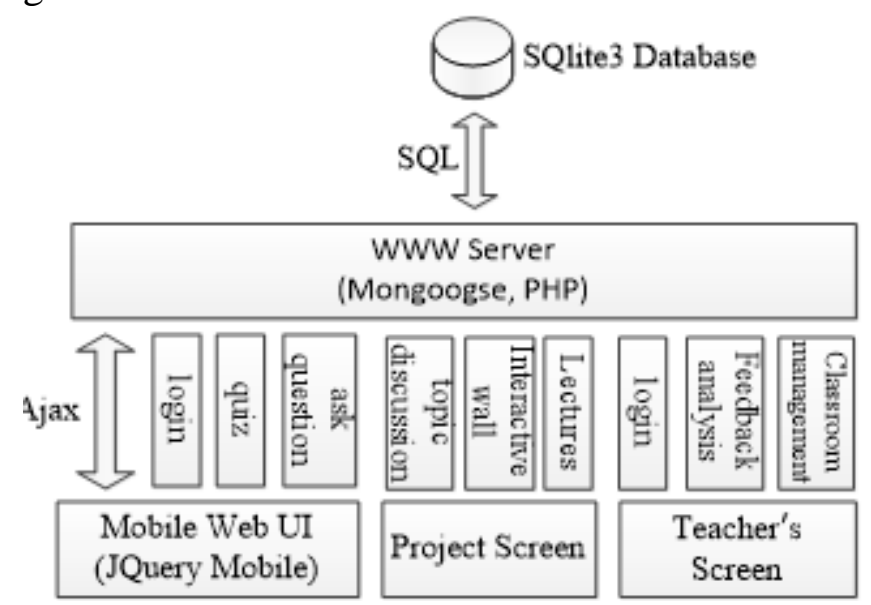

Fig. 2 Framework of the web based CIRS

Based on the feedback model, we design and realize a mobile web based classroom interactive response system (CIRS) for improving the teaching interaction in electrical and electronic course. The framework of the web based CIRS is shown in Figure 2. The whole system is configured with open source software's [5] such as Sqlite, mongoose web server and PHP server script language. Considering the universal use of smart mobile phones, we use Jquery-Mobile and Ajax to design the front-end user interface. Although the web server is configured in lightweight level, all the involved 
software's and hardware's are able to enable concurrent access from hundreds of students in a typical large scale electrical and electronic lectures. Either due to the open source web framework and the employment of smart phones, this system is easy to implement, management and maintain.

\section{Application of the feedback system in electrical and electronic course teaching}

The realized system is tested in a Xiaomi smart phone with Chrome browser and Android 4.0 operating system. Figure 3(a) shows a typical login page for the students who wish to use the system. The QR code in Fig. 3 make the access feasible in mobile platform without typing complex website address. Because of the excellent performance of Jquery mobile javascript library, the UI is accessible by a smart mobile and the students will have good use experiences.

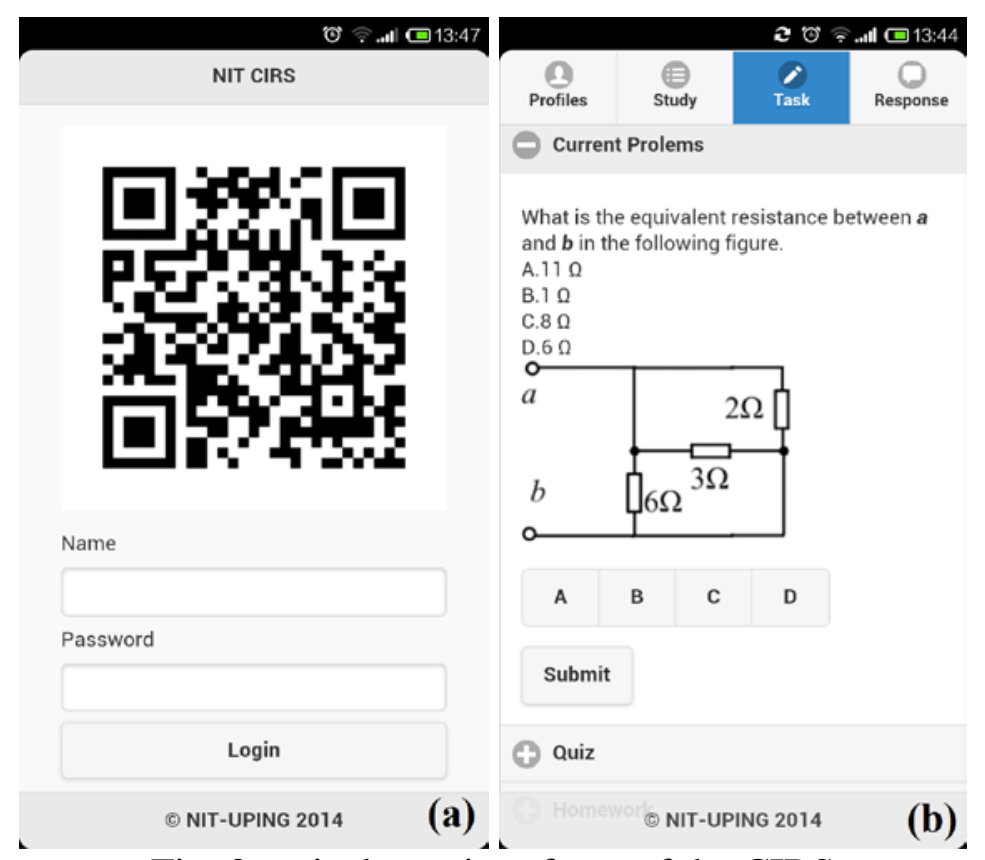

Fig. 3 typical user interfaces of the CIRS

The statistic of results of answers to the current problem:

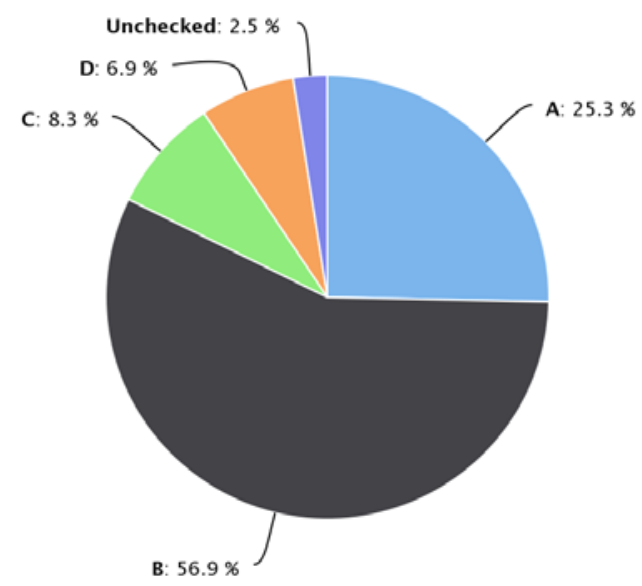

Fig. 4 Typical statistic report of the students' answers

In order to use this system in our electrical and electronic course teaching, we built quiz and question libraries of semiconductors, amplifiers circuits, feedback amplifiers, digital logical circuits and comprehensive application cases. We organize our electrical and electronic teaching with a mode named flipped classroom. So the main form of teaching in the classroom is discussion, interaction and inner construction of knowledge's. It should be noted that the students must have self-learned the 
related knowledge points before the classroom teaching. Figure 3(b) shows an example of discussion topic which the students need to decide and make a choice. In this manner, all the students can take part in the exercise through their smart mobile phones and the results can be received and evaluated in time. Then the whole analyzed results (Figure 4) are sent to the instructor for dynamic adjustment of the lecture. In our teaching experiments, we also noted that some students use their mobile phones for non-learning purpose such as surfing the Internet, play games. These issues need to be further studied and well-controlled.

\section{Summary}

In summary, we analyzed the feedback mechanism in traditional electrical and electronic lectures, and found that the feedback channel in the class is asymmetry between the instructor and the students. To resolve this issue, we proposed and designed a mobile-web-based classroom interactive response system. Through this system, the feedback information is collected and analyzed in real time, and the statistic results are sent to the teacher for dynamically controlling the teaching inertactive activity. With the prevalence of smart phone, we expect that the present CIRS will play an important role in promoting and improving the teaching of electrical and electronic courses.

\section{Acknowledgements}

This work is supported by the Teaching Reform Research Project of NIT (No. NITJG-201302), Education Scientific Planning Project of Ningbo (No. YGH039), Natural Science Funding of Ningbo (No. 2011A610107) and National Planning Project of Educational Information Technology (No. 146241691).

\section{References}

[1] Huimei Yang, Feiqing Wu, Chenyu Wu, Reform and Exploration of Electronic Basic Course Teaching, Applied Mechanics and Materials, 2014, p.1943-1946.

[2] Chenyu Wu, Wei Fang, Research of case-based teaching of electrical and electronic technology, Applied Mechanics and Materials,2014, p. 467-470.

[3] Wesley Baker. The "Classroom Flip”: Using Web Course Management Tools to Become the Guide by the Side, the 11th International Conference on College Teaching and Learning, 2000, p. 9-17.

[4] Macro Chee, Smith Le, Theory and Practice on Feedback Infromation of The contemporary Distance Education, second ed., The Central Radio and Television University Press, Beijing, 2008.

[5] Information on http://www.jquery.com, http://php.net, and http:// www.sqlite.org 